\title{
One Pile Nim with Arbitrary Move Function
}

\author{
Arthur Holshouser \\ 3600 Bullard St. \\ Charlotte, NC, USA \\ Harold Reiter \\ Department of Mathematics, \\ University of North Carolina Charlotte, \\ Charlotte, NC 28223, USA \\ hbreiter@email.uncc . edu
}

Submitted: Feb 8, 2002; Accepted: Jun 11, 2003; Published: Jul 27, 2003 MR Subject Classifications: 91A46, 11B37

\begin{abstract}
This paper solves a class of combinatorial games consisting of one-pile counter pickup games for which the maximum number of counters that can be removed on each successive move equals $f(t)$, where $t$ is the previous move size and $f$ is an arbitrary function.
\end{abstract}

The purpose of this paper is to solve a class of combinatorial games consisting of one-pile counter pickup games for which the maximum number of counters that can be removed on each successive move changes during the play of the game.

Two players alternate removing a positive number of counters from the pile. An ordered pair $(N, x)$ of positive integers is called a position. The number $N$ represents the size of the pile of counters, and $x$ represents the greatest number of counters that can be removed on the next move. A function $f: Z^{+} \rightarrow Z^{+}$is given which determines the maximum size of the next move in terms of the current move size. Thus a move in a game is an ordered pair of positions $(N, x) \mapsto(N-k, f(k))$, where $1 \leq k \leq \min (N, x)$.

The game ends when there are no counters left, and the winner is the last player to move in a game. In this paper we will consider $f: Z^{+} \rightarrow Z^{+}$to be completely arbitrary. That is, we place no restrictions on $f$. This paper extends a previous paper by the authors [2], which in turn extended two other papers, [1] and [3]. The paper by Epp and Ferguson [1] assumed $f$ is non-decreasing, and the paper [3] assumed $f$ is non-decreasing and $f(n) \geq n$. Our previous paper [2] assumed more restrictive conditions on $f$ including as a special case all $f: Z^{+} \rightarrow Z^{+}$that satisfy $f(n+1)-f(n) \geq-1$. 
The main theorem of this paper will also allow the information concerning the strategy of a game to be stored very efficiently. We now proceed to develop the theory.

Generalized Bases: An infinite strictly increasing sequence $B=\left(b_{0}=1, b_{1}, b_{2}, \cdots\right)$ of positive integers is called an infinite $g$-base if for each $k \geq 0, b_{k+1} \leq 2 b_{k}$. This "slow growth' of $B$ 's members guarantees lemma 1.

Finite $g$-bases. A finite strictly increasing sequence $B=\left(b_{0}=1, b_{1}, b_{2}, \cdots, b_{t}\right)$ of positive integers is called a finite $g$-base if for each $0 \leq k<t, b_{k+1} \leq 2 b_{k}$.

Lemma 1. Let $B$ be an infinite $g$-base. Then each positive integer $N$ can be represented as $N=b_{i_{1}}+b_{i_{2}}+\cdots+b_{i_{t}}$ where $b_{i_{1}}<b_{i_{2}}<\cdots<b_{i_{t}}$ and each $b_{i_{j}}$ belongs to $B$.

Proof. The proof is given by the following recursive algorithm. Note that $b_{0}=1 \in B$. Suppose all integers $1,2,3, \cdots, m-1$ have been represented as a sum of distinct members of $B$ by the algorithm. Suppose $b_{k} \leq m<b_{k+1}$. Then $m=\left(m-b_{k}\right)+b_{k}$. Now $m-b_{k}<b_{k}$, for otherwise, $2 b_{k} \leq m$. Since $m<b_{k+1}$, we have $2 b_{k}<b_{k+1}$, which contradicts the definition of a $g$-base. Since $m-b_{k}$ is less than $m$, it follows that $m-b_{k}$ has been represented by the algorithm as a sum of distinct members of $B$ that are less than $b_{k}$. Thus we may assume that $m-b_{k}=b_{i_{1}}+b_{i_{2}}+\cdots+b_{i_{t-1}}$ where $b_{i_{1}}<b_{i_{2}}<\cdots<b_{i_{t-1}}$ and each $b_{i_{j}}$ belongs to $B$. Then $m=b_{i_{1}}+b_{i_{2}}+\cdots+b_{i_{t}}$ where $b_{i_{t}}=b_{k}, b_{i_{1}}<b_{i_{2}}<\cdots<b_{i_{t}}$ and each $b_{i_{j}}$ belongs to $B$.

Lemma 2. Let $B=\left(b_{0}=1, b_{1}, b_{2} \cdots b_{t}\right)$ be a finite $g$-base. For any positive integer $N$, let $\theta \geq 0$ be the unique integer such that $0 \leq N-\theta b_{t}<b_{t}$. Then the same algorithm used in the proof of lemma 1 can be used to uniquely represent $N=b_{i_{1}}+b_{i_{2}}+\cdots+b_{i_{k}}+\theta b_{t}$ where $b_{i_{1}}<b_{i_{2}}<\cdots<b_{i_{k}}<b_{t}$ and each $b_{i_{j}}$ belongs to $B$.

In this paper we always use the algorithms used in the proofs of lemmas 1 and 2 to uniquely represent any positive integer $N$ as the sum of distinct members of the $g$-base that we are dealing, whether this $g$-base is finite or infinite.

Definition 3. Representation of a positive integer. Suppose $B=\left(b_{0}=1, b_{1}, b_{2}, \cdots\right)$, where $b_{0}<b_{1}<b_{2}<\cdots$, is an infinite g-base. Let $N=b_{i_{1}}+b_{i_{2}}+\cdots+b_{i_{k}}$, where $b_{i_{1}}<b_{i_{2}}<\cdots<b_{i_{k}}$, be the representation of a positive integer $N$ that is specified by the algorithm used in the proof of lemma 1 . Then we define $\bar{g}(N)=b_{i_{1}}$.

Suppose $B=\left(b_{0}=1, b_{1}, b_{2}, \cdots b_{t}\right)$, where $b_{0}<b_{1}<\cdots<b_{t}$, is a finite g-base. Let $N=b_{i_{1}}+b_{i_{2}}+\cdots+b_{i_{k}}+\theta b_{t}$, where $b_{i_{1}}<b_{i_{2}}<\cdots<b_{i_{k}}<b_{t}$ be the representation of $N$ in $B$ that is specified by the algorithm used in lemma 2. Then $\bar{g}(N)=b_{i_{1}}$ unless $N=\theta b_{t}$ in which case $\bar{g}(N)=b_{t}$.

Generating $g$-bases: For every function $f: Z^{+} \rightarrow Z^{+}$, we generate a $g$-base $B_{f}$ and a function $g^{\prime}: B_{f} \rightarrow Z^{+}$as follows.

Let $b_{0}=1, g^{\prime}\left(b_{0}\right)=1, b_{1}=2, g^{\prime}\left(b_{1}\right)=2$. Suppose $b_{0}, b_{1}, b_{2}, \cdots, b_{k}$ and $g^{\prime}\left(b_{0}\right), g^{\prime}\left(b_{1}\right)$, $g^{\prime}\left(b_{2}\right), \cdots, g^{\prime}\left(b_{k}\right)$, where $k \geq 1$, have been generated. Then $b_{k+1}=b_{k}+b_{i}$ where $b_{i}$ is the smallest member of $\left\{b_{0}, b_{1}, \cdots, b_{k}\right\}$ such that $g^{\prime}\left(b_{i}\right)=b_{i}$ and $f\left(b_{i}\right) \geq g^{\prime}\left(b_{k}\right)$ if such a $b_{i}$ exists. If no such $b_{i}$ exists for some $k$, the $g$-base $B_{f}$ is finite. Also, $g^{\prime}\left(b_{k+1}\right)=$ 
$\min \left[\left\{b_{k+1}\right\} \cup\left\{b_{k+1}-b_{k}+\bar{x}: 1 \leq \bar{x}<b_{k}\right.\right.$ and $\left.\left.f\left(b_{k+1}-b_{k}+\bar{x}\right)<g^{\prime}\left(\bar{g}\left(b_{k}-\bar{x}\right)\right)\right\}\right]$. Of course, $\bar{g}\left(b_{k}-\bar{x}\right)$ is computed using definition 3 with $\left(b_{0}=1, b_{1}, b_{2}, \cdots, b_{k}\right)$, and min $S$ means the smallest member of $S$. We will explain later why $B_{f}$ and $g^{\prime}$ are defined this way. Also, we note that since $b_{k+1}=b_{k}+b_{i}$ and $b_{0}=1 \leq b_{i} \leq b_{k}$, it follows that $B_{f}$ is indeed a $g$-base.

Definition 4. Suppose $f: Z^{+} \rightarrow Z^{+}$generates the g-base $B_{f}$ and the function $g^{\prime}: B_{f} \rightarrow$ $Z^{+}$. Then for every $N \in Z^{+}$, we define $g^{\prime}(N)=g^{\prime}(\bar{g}(N))$, where $\bar{g}(N)$ is computed using $B_{f}$. Thus in the definition of $g^{\prime}\left(b_{k+1}\right)$, we could substitute $g^{\prime}\left(b_{k}-\bar{x}\right)$ for $g^{\prime}\left(\bar{g}\left(b_{k}-\bar{x}\right)\right)$.

Before we state the main theorem, we need a few more definitions. We recall that for our game, we are given some arbitrary function $f: Z^{+} \rightarrow Z^{+}$. That is, we place no restrictions on $f$. Also, a position in the game is an ordered pair of positive integers $(N, x)$, and a move is $(N, x) \mapsto(N-k, f(k)), 1 \leq k \leq \min (N, x)$. A position $(N, x)$ is called unsafe if it is unsafe to move to it. Since the player who moves to an unsafe position loses with best play, the player who moves from an unsafe position can always win. Similarly, a position is safe if it is safe to move to it. For each ordered pair $(N, x)$, define $F(N, x)=0$ if $(N, x)$ is a safe position, and $F(N, x)=1$ if $(N, x)$ is an unsafe position. Note that $F(N, x)=1$ when the list $F(N-1, f(1)), F(N-2, f(2)), \cdots, F(N-x, f(x))$ contains at least one 0 . Note that $F(0, x)=0$ for all $x \in Z^{+} . F(N, x)=0$ when this list contains no 0 's. We imagine that $F(1, x), x=1,2, \cdots$, is computed first. Then $F(2, x), x=1,2,3, \cdots$, is computed. Then $F(3, x), x=1,2,3, \cdots$, is computed, etc. Note that $F(N, x)=1$ when $N \leq x$. Note also that for a fixed $N \in Z^{+}$and a variable $x \in Z^{+}$, the infinite sequence $F(N, x), x=1,2,3, \cdots$, always consists of a finite string (possibly empty) of consecutive 0 's followed by an infinite string of consecutive 1's. This is because $F(N, x)=1$ when $x \geq N$ and also once the sequence first switches from 0 to 1 it must retain the value of 1 thereafter. For each $N \in Z^{+}$, define $g(N)$ to be the smallest $x \in Z^{+}$such that $F(N, x)=1$. Of course $1 \leq g(N) \leq N$. For every $N \in Z^{+}, g(N)$ is the position of the first 0 in the sequence $F(N-1, f(1)), F(N-2, f(2)), \cdots, F(N-g(N), f(g(N))$. This means that $F(N-g(N), f(g(N)))=0$, but all preceding members of this sequence have a value of 1 . It is obvious that $F(N, x)=0$ when $x \leq g(N)-1$ and $f(N, x)=1$ when $x \geq g(N)$. We can now state the main theorem.

Main theorem: Suppose we play our game with an arbitrary but fixed move function $f: Z^{+} \rightarrow Z^{+}$. Suppose $f$ generates the $g$-base $B_{f}$ and the function $g^{\prime}: B_{f} \rightarrow Z^{+}$. Then for every $N \in Z^{+}, g(N)=g^{\prime}(N)$ where $g^{\prime}(N)$ is defined in definition 4 using the $g$-base $B_{f}$ and the function $g^{\prime}: B_{f} \rightarrow Z^{+}$.

The main theorem implies that a position $(N, x)$ is unsafe if $x \geq g^{\prime}(N)$ and safe if $x<g^{\prime}(N)$. This means a winning move must be $(N, x) \rightarrow\left(N-g^{\prime}(N), f\left(g^{\prime}(N)\right)\right)$. The reader will note that the theorem is true whether $B_{f}$ is finite or infinite. In a moment we will write a detailed proof for the case where $B_{f}$ is infinite. The proof of the finite case, which involves a slight modification of the infinite case, is left to the reader.

Before we begin the proof, we would like to point out that for an enormous number of functions $f$ it is very easy to compute $B_{f}$ and $g^{\prime}$. For example, if $f$ is non-decreasing or if $f$ satisfies $f(n+1)-f(n) \geq-1$, then $g^{\prime}: B_{f} \rightarrow Z^{+}$is just the identity function on $B_{f}$, and $B_{f}$ is generated by the following very simple algorithm. $b_{0}=1, b_{1}=2$ and if 
$b_{0}, b_{1}, b_{2}, \cdots, b_{k}$ have been generated, then $b_{k+1}=b_{k}+b_{i}$ where $b_{i}$ is the smallest member of $\left\{b_{0}, b_{i}, \cdots b_{k}\right\}$ such that $f\left(b_{i}\right) \geq b_{k}$. There are also many other functions $f$ for which $B_{f}$ and $g^{\prime}$ are very easy to compute. However, for many functions $f$, the best way to compute $B_{f}$ and $g^{\prime}$ is to go ahead and compute $g(N)$ first. Note that $g(N), \quad N=1,2,3, \cdots$, can be computed directly by the following algorithm: $g(1)=1, g(2)=2$. If $g(1), g(2), \cdots, g(k-$ $1), k \geq 3$, have been computed, then $g(k)$ is the smallest $x \in\{1,2,3, \cdots, k\}$ such that $f(x)<g(k-x)$, where we agree that $g(0)=\infty$.

For those functions where $g(N)$ is computed first, it may appear that this paper has no advantages whatsoever since we already know $g(N)$. However, once $g(N)$ is computed, we know that $g^{\prime}=g$, and we can then easily compute $B_{f}$.

Once we know $B_{f}$ and $g^{\prime}: B_{f} \rightarrow Z^{+}$, we know that $B_{f}$ and $g^{\prime}$ by themselves store the complete strategy of the game. Quite often the members of $B_{f}$ grow exponentially. In these cases we have an efficient way of storing the strategy of the game. We conjecture that if $g$ is unbounded, then the members of $B_{t}$ always grow exponentially.

At the end of this paper, we give two examples in which $B_{f}, g^{\prime}$ provide efficient storage. We will also give an example when $B_{f}, g^{\prime}$ provide inefficient storage. In the first two examples, $g$ is unbounded and in the third, $g$ is bounded.

Proof. The main theorem follows easily if we can prove the following statements. We assume $B_{f}$ is infinite.

1. $\forall b_{i} \in B_{f}, g\left(b_{i}\right)=g^{\prime}\left(b_{i}\right)$,

2. $\forall N \in Z^{+} \backslash B_{f}, g(N)<N$,

3. $\forall N \in Z^{+} \backslash B_{f}$, if $b_{i}<N<b_{i+1}$, then $N=b_{i}+\left(N-b_{i}\right)$, where $1 \leq N-b i<b_{i}$, and $g(N)=g\left(N-b_{i}\right)$. Of course, $1 \leq N-b_{i}<b_{i}$ is obvious since $B_{f}$ is a $g$-base.

Note: At the end of the proof, we will use property 3 to explain why we defined $B_{f}$ the way that we did.

The main theorem follows because if $N=b_{i_{1}}+b_{i_{2}}+\cdots+b_{i_{k}}$, where $b_{i_{1}}<b_{i_{2}}<\cdots<b_{i_{k}}$, is the representation of $N$ in the infinite $g$-base $B_{f}$ that is computed by the algorithm used in the proof of lemma 1 , we have the following.

$$
\begin{aligned}
g(N) & =g\left(\left(b_{i_{1}}+\cdots+b_{i_{k-1}}\right)+b_{i_{k}}\right)=g\left(\left(b_{i_{1}}+\cdots+b_{i_{k-2}}\right)+b_{i_{k-1}}\right) \\
& =g\left(\left(b_{i_{1}}+\cdots+b_{i_{k-3}}\right)+b_{i_{k-2}}\right)=\cdots= \\
& =g\left(b_{i_{1}}\right)=g^{\prime}\left(b_{i_{1}}\right)=g^{\prime}(N),
\end{aligned}
$$

by the definition of $g^{\prime}(N)$ since $g\left(b_{i_{1}}\right)=g^{\prime}(\bar{g}(N))$.

Note that once we have proved statements $1,2,3$ for all members of $\{1,2,3, \cdots, \bar{k}\}$, then $\forall N \in\{1,2,3, \cdots \bar{k}\}, g(N)=g^{\prime}(N)$ will also be true.

We prove statements $1,2,3$ by mathematical induction. First, note that no matter what $f$ is $g(1)=g^{\prime}(1)=1$ and $g(2)=g^{\prime}(2)=2$. Conditions 2,3 do not apply for integers 1,2 since $\{1,2\} \subseteq B_{f}$. 
Let us suppose that condition 1 is true for all $b_{i} \in\left\{b_{0}, b_{1}, b_{2}, \cdots, b_{k}\right\}$, where $k \geq 1$, and conditions 2,3 are true for all $N \in\left\{1,2,3,4, \cdots, b_{k}\right\} \backslash B_{f}$. We show that conditions 2,3 are true for all $N \in\left\{b_{k}+1, b_{k}+2, \cdots, b_{k+1}-1\right\}$, and condition 1 is true for $b_{i}=b_{k+1}$. Define $b_{\theta(k)}$ by $b_{k+1}=b_{k}+b_{\theta(k)}$, where $b_{\theta(k)} \in\left\{b_{0}, b_{1}, b_{2}, \cdots, b_{k}\right\}$.

In the following argument, we omit the first part when $b_{k+1}-b_{k}=1$. So let us imagine that $b_{k+1}-b_{k} \geq 2$. We will prove that conditions 2,3 are true for $N \in\left\{b_{k}+\right.$ $\left.1, b_{k}+2, \cdots, b_{k+1}-1\right\}$ by proving this sequentially with $N$ starting at $N=b_{k}+1$ and ending at $N=b_{k+1}-1$.

Note that once we prove condition 3 for any $N \in\left\{b_{k}+1, \cdots, b_{k+1}-1\right\}$, condition 2 will follow for this $N$ as well. This is because if $N=b_{k}+\left(N-b_{k}\right)$, where $1 \leq N-b_{k}<b_{k}$, and $g(N)=g\left(N-b_{k}\right)$, then $g(N)=g\left(N-b_{k}\right) \leq N-b_{k}<N$. Note that $g\left(N-b_{k}\right) \leq N-b_{k}$ is always true. So let us now prove condition 3 is true for $N$ as $N$ varies sequentially over $b_{k}+1, \cdots, b_{k+1}-1$.

Since we are assuming that $b_{k+1}-b_{k} \geq 2$, this means that $f(1)<g^{\prime}\left(b_{k}\right)=g\left(b_{k}\right)$ is assumed as well since $g^{\prime}(1)=1$. Therefore, $g\left(b_{k}+1\right)=g(1)=1$ is obvious. Therefore, suppose we have proved condition 3 for all $N \in\left\{b_{k}+1, b_{k}+2, \cdots, b_{k}+t-1\right\}$ where $b_{k}+t-1 \leq b_{k+1}-2$. This implies for all $N \in\left\{1,2,3, \cdots, b_{k}+t-1\right\}, g(N)=g^{\prime}(N)$. We now prove condition 3 for $N=b_{k}+t$. This means we know that $g(i)=g\left(b_{k}+i\right), i=$ $1,2,3, \cdots, t-1$ and we wish to prove $g(t)=g\left(b_{k}+t\right)$. Recall that $g(t)$ is the smallest positive integer $x$ such that the list (1) $F(t-1, f(1)), F(t-2, f(2)), \cdots, F(t-x, f(x))$ contains exactly one 0 (which comes at the end of the list).

Also, $g\left(b_{k}+t\right)$ is the smallest positive integer $x$ such that the list (2) $F\left(b_{k}+t-\right.$ $1, f(1)), F\left(b_{k}+t-2, f(2)\right), \cdots, F\left(b_{k}+t-x, f(x)\right)$ contains exactly one 0 (which comes at the end). Since we are assuming that $g(i)=g\left(b_{k}+i\right), i=1,2, \cdots, t-1$, we know that the above two lists must be identical as long as $1 \leq x \leq t-1$. This follows from the definition of $g$ since $g(N)$ tells us that $F(N, x)=0$ when $1 \leq x \leq g(N)-1$ and $f(N, x)=1$ when $g(N) \leq x$. Now if $t \notin\left\{b_{0}=1, b_{1}, b_{2} \cdots, b_{\theta(k)-1}\right\}$, we know from condition 2 that $g(t)<t$. This tells us that for list (1) the smallest $x$ such that list (1) contains exactly one 0 satisfies $1 \leq x \leq t-1$. Therefore, since the two lists (1),(2) are identical when $1 \leq x \leq t-1$, this tells us that $g(t)=g\left(b_{k}+t\right)$.

Next, suppose $t \in\left\{b_{0}=1, b_{1}, b_{2} \cdots, b_{\theta(k)-1}\right\}$. Of course, $g(t)=g^{\prime}(t)$ since $t<b_{k}+t-1$. Now if $g(t)=g^{\prime}(t)<t$, the same argument used above holds to show that $g(t)=g\left(b_{k}+t\right)$. Now if $g(t)=g^{\prime}(t)=t$, we know from the definition of how $b_{k+1}=b_{k}+b_{\theta(k)}$ is generated that $f(t)<g^{\prime}\left(b_{k}\right)=g\left(b_{k}\right)$. Since $g^{\prime}(t)=g(t)=t$, we know that the first $t-1$ members of each of the lists (1), (2) consists of all 1's since they are identical up this point and $g(t)=t$. Now in list $(1), F(t-t, f(t))=F(0, f(t))=0$. Also, in list $(2), F\left(b_{k}+t-t, f(t)\right)=$ $F\left(b_{k}, f(t)\right)=0$ since $f(t)<g\left(b_{k}\right)=g^{\prime}\left(b_{k}\right)$. This tells us that $g(t)=g\left(b_{k}+t\right)=t$ when $t \in\left\{b_{0}, b_{1}, \cdots, b_{\theta(k)-1}\right\}$, and $g(t)=g^{\prime}(t)=t$. Of course, $g(t)=g\left(b_{k}+t\right)$ is what we wished to show.

Finally, we show that $g\left(b_{k+1}\right)=g^{\prime}\left(b_{k+1}\right)$. Recall that $b_{k+1}=b_{k}+b_{\theta(k)}$ where $g^{\prime}\left(b_{\theta(k)}\right)=$ $g\left(b_{\theta(k)}\right)=b_{\theta(k)}$ and $f\left(b_{\theta(k)}\right) \geq g^{\prime}\left(b_{k}\right)=g\left(b_{k}\right)$. We now know that $\forall N \in\left\{1,2,3,4, \cdots, b_{k+1}-\right.$ $1\}, g(N)=g^{\prime}(N)$. Also, we know that $g(i)=g\left(b_{k}+i\right), i=1,2,3, \cdots, b_{\theta(k)}-1$. Since $g\left(b_{\theta(k)}\right)=b_{\theta(k)}$ we know that all terms in the following sequence are 1's except the final 
term which is 0 .

(3) $F\left(b_{\theta(k)}-1, f(1)\right), F\left(b_{\theta(k)}-2, f(2)\right), F\left(b_{\theta(k)}-3, f(3)\right), \cdots, F\left(1, f\left(b_{\theta(k)}-1\right)\right)$, $F\left(0, f\left(b_{\theta(k)}\right)\right)=0$. Now $g\left(b_{k+1}\right)=g\left(b_{k}+b_{\theta(k)}\right)$ is the position of the first 0 in the following sequence where we note that the position of a term $F\left(b_{k}+b_{\theta(k)}-i, f(i)\right)$ is $i$.

(4) $F\left(b_{k}+b_{\theta(k)}-1, f(1)\right), F\left(b_{k}+b_{\theta(k)}-2, f(2)\right), \cdots, F\left(b_{k}+1, f\left(b_{\theta(k)}-1\right)\right), F\left(b_{k}, f\left(b_{\theta(k)}\right)\right)$, $F\left(b_{k}-1, f\left(b_{\theta(k)}+1\right)\right), \quad F\left(b_{k}-2, f\left(b_{\theta(k)}+2\right)\right), \cdots, F\left(1, f\left(b_{\theta(k)}+b_{k}-1\right)\right), F\left(0, \overline{\left.f\left(b_{\theta(k)}+b_{k}\right)\right)}=\right.$ 0 .

Since $g\left(b_{k}+i\right)=g(i), i=1,2, \cdots, b_{\theta(k)}-1$, we know that the first $b_{\theta(k)}-1$ terms of (4) are 1's since the first $b_{\theta(k)}-1$ terms of (3) are 1's. Now $F\left(b_{k}, f\left(b_{\theta(k)}\right)\right)=1$ from the definition of $g$ since $f\left(b_{\theta(k)}\right) \geq g^{\prime}\left(b_{k}\right)=g\left(b_{k}\right)$. Note that the last term in (4) is 0 . Recall that for all $N \in\left\{1,2,3, \ldots, b_{k+1}-1\right\}, \quad g(N)=g^{\prime}(N)$.

From (4), we see that $g\left(b_{k+1}\right)$ is the smallest $b_{\theta(k)}+\bar{x}, 1 \leq \bar{x} \leq b_{k}$ such that $F\left(b_{k}-\right.$ $\left.\bar{x}, f\left(b_{\theta(k)}+\bar{x}\right)\right)=0$. Since $F\left(b_{k}-b_{k}, f\left(b_{\theta(k)}+b_{k}\right)\right)=0$, we see that $g\left(b_{k+1}\right)$ is the smaller of $b_{\theta(k)}+b_{k}=b_{k+1}$ and the smallest $b_{\theta(k)}+\bar{x}, 1 \leq \bar{x}<b_{k}$, such that $F\left(b_{k}-\bar{x}, f\left(b_{\theta(k)}+\bar{x}\right)\right)=0$ if such a $b_{\theta(k)}+\bar{x}$ exists. Now $F\left(b_{k}-\bar{x}, f\left(b_{\theta(k)}+\bar{x}\right)\right)=0$, when $1 \leq \bar{x}<b_{k}$, if and only if $f\left(b_{\theta(k)}+\bar{x}\right)<g\left(b_{k}-\bar{x}\right)$. Since $b_{\theta(k)}=b_{k+1}-b_{k}$ and since $g\left(b_{k}-\bar{x}\right)=g^{\prime}\left(b_{k}-\bar{x}\right)=$ $g^{\prime}\left(\bar{g}\left(b_{k}-\bar{x}\right)\right)$, if we compare the above definition of $g\left(b_{k+1}\right)$ with the definition of $g^{\prime}\left(b_{k+1}\right)$ given earlier in this paper, we see that $g\left(b_{k+1}\right)=g^{\prime}\left(b_{k+1}\right)$.

Observation: We now explain why we defined $B_{f}$ the way that we did. Assuming that $B_{f}$ is infinite, we know from the definition of $B_{f}$ that $b_{i+1}-b_{i} \leq b_{i}$.

Also, from the definition of $b_{i+1}$, we know that $g^{\prime}\left(b_{i+1}-b_{i}\right)=b_{i+1}-b_{i}$.

Also, from the definition of $g^{\prime}\left(b_{i+1}\right)$ (since $\bar{x} \geq 1$ in the definition), we know that $g^{\prime}\left(b_{i+1}\right)>b_{i+1}-b_{i}=g^{\prime}\left(b_{i+1}-b_{i}\right)$. Thus $g\left(b_{i+1}\right)>g\left(b_{i+1}-b_{i}\right)$.

However, from statement 3 (at the beginning of the proof) we know that for all $N$ satisfying $b_{i}<N<b_{i+1}$ it is true that $g(N)=g\left(N-b_{i}\right)$. This change at $b_{i+1}$ is precisely why we defined $B_{f}$ the way that we did.

The misère version: To win at the misère version $(N, x)$ of dynamic nim, simply use the theory to win the game $(N-1, x)$, so that your opponent is forced to take the last counter. The reader may like to figure out the strategy for the following variation. Suppose $S \subseteq Z^{+} \cup\{0\}$, and the game is over as soon as $N \in S, N$ being the pile size. In this paper $S=\{0\}$.

A difficult problem is to find (with proof) functions $f: Z^{+} \rightarrow Z^{+}$such that $B_{f}$ and $g^{\prime}$ satisfy the following. $\left\{b_{i}: b_{i} \in B_{f}, g^{\prime}\left(b_{i}\right)<b_{i}\right\}$ is infinite and $\left\{b_{i}: b_{i} \in B_{f}, g^{\prime}\left(b_{i}\right)=b_{i}\right\}$ is infinite. We now give two examples of such functions. In both examples, $B_{f}$ and $g^{\prime}$ will store the strategy extremely efficiently since the members of $B_{f}$ grow exponentially.

Example 1: Define $f(n)=n, n \neq 8^{k}, k=0,1,2,3, \cdots, f\left(8^{k}\right)=4 \cdot 8^{k}$.

Then $B_{f}=\left\{a \cdot 8^{b}: a, b\right.$ integers, $\left.1 \leq a \leq 7,0 \leq b\right\}$, and $g^{\prime}\left(a \cdot 8^{b}\right)=\phi(a) \cdot 8^{b}$, where $\phi(1)=1, \phi(2)=2, \phi(3)=3, \phi(4)=4, \phi(5)=2, \phi(6)=2, \phi(7)=3$.

Noting that $g^{\prime}\left(a \cdot 8^{b}\right) \leq 4 \cdot 8^{b}$, we leave the proof as an exercise for the reader. The proof is by induction in blocks of seven starting with proving that $\{1,2,3,4,5,6,7\} \subseteq B_{f}$ and $g^{\prime}(1)=1, g^{\prime}(2)=2, g^{\prime}(3)=3, g^{\prime}(4)=4, g^{\prime}(5)=2, g^{\prime}(6)=2, g^{\prime}(7)=3$. 
Example 2: Define $f(n)=n$, if $n$ is even and $f(n)=4 n$, if $n$ is odd.

Instead of calling $B_{f}=\left(b_{0}=1<b_{1}<b_{2}<\cdots\right)$, it is more convenient to call $B_{f}=$ $\left(a_{1}=1<b_{1}<c_{1}<a_{2}<b_{2}<c_{2}<d_{2}<a_{3}<b_{3}<c_{3}<d_{3}<\cdots<a_{i}<b_{i}<c_{i}<d_{i}<\right.$ $\cdots)$. The first few terms of $B_{f}$ and the corresponding $g^{\prime}$ are computed by the following recursion. First, we define a strictly increasing sequence $\Delta_{2}, \Delta_{3}, \ldots$ recursively as follows: $\Delta_{2}=1, \Delta_{3}=3$ and for all $i \geq 4, \Delta_{i}=\Delta_{i-1}+4 \Delta_{i-2}$. Also, define $a_{1}=1, g^{\prime}\left(a_{1}\right)=1, b_{1}=$ $2, g^{\prime}\left(b_{1}\right)=2, c_{1}=3, g^{\prime}\left(c_{1}\right)=3, a_{2}=4, g^{\prime}\left(a_{2}\right)=4, b_{2}=5, g^{\prime}\left(b_{2}\right)=2, c_{2}=6, g^{\prime}\left(c_{2}\right)=$ $2, d_{2}=7, g^{\prime}\left(d_{2}\right)=7$. For all $i \geq 3$, define $a_{i}, g^{\prime}\left(a_{i}\right), b_{i}, g^{\prime}\left(b_{i}\right), c_{i}, g^{\prime}\left(c_{i}\right), d_{i}, g^{\prime}\left(d_{i}\right)$ recursively as follows: $a_{i}=d_{i-1}+\Delta_{i}, g^{\prime}\left(a_{i}\right)=a_{i}, b_{i}=a_{i}+\Delta_{i}, g^{\prime}\left(b_{i}\right)=2 \Delta_{i}, c_{i}=b_{i}+\Delta_{i}, g^{\prime}\left(c_{i}\right)=$ $2 \Delta_{i}, d_{i}=c_{i}+\Delta_{i}, g^{\prime}\left(d_{i}\right)=d_{i}$.

In the third example, we give an example of a function $f$ such that $B_{f}=Z^{+}$. If $B_{f}=Z^{+}$, it is easy to prove that $g$ must be bounded. The reader can show that if either $g$ or $f$ is bounded, then eventually $g$ must become periodic.

Example 3: Let $f(1)=4, f(n)=2, n \geq 2$. Then $g(1)=1, g(2+4 k)=2$, $g(3+4 k)=3, g(4+4 k)=4, g(5+4 k)=2, k=0,1,2,3, \cdots$. Also, it is easy to see that $B_{f}=Z^{+}$. Of course, $g^{\prime}=g$ on $B_{f}$.

Appendix. The functions $f, g, g^{\prime}$ and the base $B_{f}$ described in this paper have a great many properties, a few of which are listed below. Recall that $f$ is given, and it determines the other three.

1. $g$ is periodic on $Z^{+}$if and only if $B_{f}$ is finite.

2. If $f$ is bounded, there exists a positive integer $a$ such that $g$ is periodic on $[a, \infty)$.

3. If $g$ is bounded, then there exists a positive integer $a$ such that $g$ is periodic on $[a, \infty)$.

4. Suppose the positive integer $a$ satisfies $f(i)<g(a)$ for all $i=1,2,3, \ldots, a$. Then $g$ is periodic on $Z^{+}$and $[1, a]$ is a period.

5. $g$ is bounded if and only if $\left\{b_{i}: b_{i} \in B_{f}, g\left(b_{i}\right)=b_{i}\right\}$ is finite.

6. There exists a positive integer $a$ such that $g$ is periodic on $[a, \infty)$ if and only if $g$ is bounded on $B_{f}$.

Acknowledgement. The authors also acknowledge the referee who made some timely and valuable suggestions. 


\section{References}

[1] Richard Epp and Thomas Ferguson, A Note on Takeaway Games, The Fibonacci Quarterly, 18 (1980), 300-303.

[2] A. Holshouser, J. Rudzinski and H. Reiter, Dynamic One-Pile Nim, The Fibonacci Quarterly, to appear.

[3] A. J. Schwenk, Take-Away Games, The Fibonacci Quarterly, 8 (1970), 225-234. 